necessary to acquire the habit of noting down on the spot, or at least on the same day, the details of a case; yet this occupies little time, and this supplement to memory, or to adopt Addington Symonds's phrase this "mecharrical memory," may in future emergencies-for example, legal proceedings, or certificates-be of the greatest use. In addition to writing a little every day some time should be given each day to reading, both of a professional and of a general kind, so as to keep up to date and maintain the general culture so essential to the medical practitioner, whose motto should be "Nihil humanum a me alienum puto." As an element in success accuracy and punctuality should not be overlooked; a present doyen of the profession when a young man on arriving at one of his first consultations was met by a senior surgical colleague standing watch in hand on the doorstep and the remark that a habit of being five minutes late boded badly for future success; this hint, gentle c. otherwise, had a lasting effect and the resulting meticulous punctuality no doubt played some part in his triumphal career. A more difficult factor in avoiding failure is to be prepared for whatever emergencies or calls may occur in practice; this demands thoughtful foresight and some imagination. The whims of ehance of course influence the future in ways that to some onlookers may seem even unfair, but probably many of the "lucky ones" are so in virtue of their ability to turn opportunities as they arise into solid advantages.

Though this address is headed "The Problem of Snecess for Medical Women," at this comparatively early stage of women's activity in the medical profession it is not wise, and indeed is hardly possible, to say the last word cin this subject. That medical women have succeeded in the face of great difficulties is shown by recent history, and now that circumstances have been so vastly improved by the pioneers the road has been opened to their successors. The way, no doubt, is long and beset by difficulties; patience, perseverance, and wisdom are necessary though exacting requisites, but there is encouraging evidence that by these means women may look forward to a just meed of success.

'Smith, Sydney : Edinburgh Review, 1810, $2 \times x$. 'Ludoviei, L. Woman, A Findication, p. 359. 1923.

\section{THE REGULATING AND REFLEX PROCESS."}

BY

P. T. HERRING, M.D.,

PBOFESSOR OF PHYSTOLOGY, ST. ANDREWS UNIVERSITY, ARD

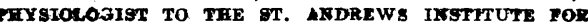
CLINICAL RESThECH.

\section{Pant I.-THE ANATOMICAI AND PHYsiological UNITS.}

That the early symptoms of disease manifest themselves $k y$ alterations in the normal reflexes of the body is a postulate laid down by Sir James Mackenzie. The truth or otherwise of this hypothesis is being investigated at the St. Andrews Institute for Clinical Research, but before any steps can be taken in its application to the study of abnormal conditions it is essential that a clear understanding be arrived at of what the normal reflex really signifies, and of the general principles which govern its production. Probably no branch of physiology has made greater progress in recent years than that relating to the nerrons system. Many problems still await solution, but general prineiples are emerging which are of far-reaching importance.

A brief review of the general principles underlying reflex action is essayed in these articles, and an attempt is marte to extend their application to the study of abnormal conditions. The author is conscious that some of his interpretations of ascertained facts are open to criticism, and may be modified in the course of time. There can be no finality in a subject of this importance, but a statement of the general position as it seems at present to members of the St. Andrews Institute may be of value.

* The expense of this research was in part met by a contribution from the Medical Research Councll.
The ANatomical Unit.

The anatomical unit of the nervous system is the neurone, which is the nerve cell with its processes, dendrites, and axis cylinder. Chains of such units make up the nerrous system. The neurones do not blend with one another anatomically at their junctions but are separated by a minute interspace in which there is a material endowed with distinct and highly important properties. This is the synapse or " synaptic nembrane."

\section{The Reflex.}

It is improbable that any one anatomical nnit in the nervous system can under normal conditions function by itself in the transmission of nerve impulses. For most purposes at least two neurones with an intervening synapse appear to be necessary to make up a functional unit. One of these neurones must be an afferent one whose province it is to transmit the nerve impulses excited in it by the stimulation of specialized end-organs or " receptors." The other neurone is an efferent one, and conducts the nerve impulses to some tissue or organ, which responds by whatever action characterizes it. Between the reacting organ or " effector" and the ending of the efferent neurone there is another minute interval oceupied by a material somewhat similar in its properties to that of the synapse. Such on arrangenent of anatomical units forms the basis of the simplest functional unit, which is the "reffex." An apparent exception to this statement is seen in the case of the so-called "axon reflex," where an afferent neurone sends branches to neighbouring arterioles and capillaries. But in the axon reflex the nerve impulses arise as usual at the receptors and pass to the central nerrous system as well as to the bloed vessels. The axon reflex appears to be a complete physiological unit in that a suitable stimulus applied to the skin produces a local vaso-dilatation of the blood ressels by the passage of nerve impulses over part of the afferent neurone only. But we do not know if this is the sole result of the stimulus, for the same neurone conducts the nerve impulses to the central nerrous system, and sensation and reflex action may result in addition to the axon reflex. The axon reflex illustrates a principle of fundamental importance, because it shows that nerve impulses set up by a receptor in an afferent neurone may be productive of more than one kind of end-result. In general it may be said that the reflex is the physiological or functional unit, and that the reactions of the nervous system are built ap of such units.

The modern view of the function of the neurone is that it conducts nerve impulses. It does so in response to stimulation, just as a muscle cell develops a state of tersion in response to stimulation. But the nervous system is more than a highly elaborate conducting mechanism. The neurome conducts, but it manifests some degree of ener in doing so, though the amount of that energy is very smail. There is no eridenee that under normal conditions the nerre impulse dies down or undergoes a decrement in the neurone. The nerve impulse may be extingwished in the central nervons system, bat such extinction always takes place at the junctions between the neurones-namely, in the synapses-and not in the neurone itself. On the other hand, nerve impulses set up in an afferent neurone may spread widely in the central nervous system, and the total sum of the resulting discharges by the efferent neurones may be greatly increased.

\section{Rhythmical Activity of the Neurone.}

Single, unrepeated nerve impulses do not, as such, find a passage through the central nervous system. In the quickest voluntary movement possible the muscular contraction is due to more than one nerve impulse, and there is evidence that the efferent neurones supplying voluntary muscle have a rhythm of discharge of about 50 per second. It is not known whether all neurones possess the property of rhythmical discharge, or what differences occur among them. It has been shown by Sherrington that if a stimulus applied to the afferent neurone in a reflex arc is sufficiently strong the efferent neurone has an afterdischarge, and the muscle supplied by it remains in activity 
after the stimulus has ceased for a period depending upon the intensity of the stimulus to the afferent neurone. The rhythm in the efferent neurone is initiated by nerve impulses from the afferent side, but persist for some time after the original stimulus has ceased.

The evidence, therefore, is in favour of rhythmical activity being a property of certain neurones-for example, efferent neurones. The possession of rhythmical activity by the neurone would be disastrous to the orderly working of the nervous system were it not for the property of inhibition by which the discharge of nerve impulses can be immediately checked when their continuance would interfere with other reactions. This property of inhibition appears to reside in the synapses, and can be brought into action in several ways.

We do not know if rhythmical activity is a property of the afferent neurones or of the receptors which initiate the nerve impulses in them. A certain amount of persistence of some sensory impressions does occur, but varies considerably in different sensations. In the sense of hearing the receptors in the cochlea are stimulated by mechanical means, the principle of sympathetic vibration being made use of. If the receptors respond to each individual vibration a series of impulses would be set up in the auditory nerve; but we do not know if this is the case. A damping mechanism is described, which would serve to cut short the response of the receptor. Mechanical vibrations can be distinguished by other parts of the body, and the impressions are discrete even with a high rate of frequency, a fact which militates against tho possession of rhythmical activity by the afferent neurones concerned. In receptors which are excited by chemical means-for example, the senses of smell and possibly of vision-the sensation is more persistent. The persistence of sensation, however, may be due to other causes than a continued action of the receptors, and may be of central production. The amount of rhythmical action possessed by the different neurones of the nervous system is an important question, but it is difficult to see how it is to be determined, and for the present it lies in the realms of hypothesis only.

Automaticity of the Neurone.

It has long been debated whether nerve cells possess the property of automaticity. Some neurones certainly do not. It would be a catastrophe if the afferent neurones possessed the power of initiating nerve impulses on their own account; all kinds of sensory illusions would be of constant occurrence. Sensory phenomena are common in disease and may be ascribed to several causes. If due to the activity of afferent neurones some form of stimulation extraneous to the neurone is the exciting cause. Normally there is no evidence that the afferent neurones conduct any impulses other than those which have originated in the receptors at the periphery. Nor is it likely that efferent cells are endowed with automaticity, though attempts have been made to show that some of them do act in this manner. The efferent nerve cells supplying the muscles of respiration have an action that appears to be automatic, but it is impossible to cut off from them all afferent impulses. Further, the respiratory centre is a complex, and some part of it is susceptible to hydrogen-ion changes in the tissue fluid in its vicinity, but precise information is lacking as to where the action takes place. Haldane states that " to judge from analogy the sensitive elements are probably not the bodies of nerve cells, but end-organs or arborizations."

We have little evidence that chemical substances, except in gross amounts, act directly upon the neurone, whereas the synapse or junction between the neurones is particularly susceptible to even minute amounts of certain chemical agents. It is possible that the action takes place at the synapses in the respiratory centre, and that some of these are specially sensitive to changes in the hydrogen ion. Any other explanation would require the interposition, somewhere in the respiratory centre, of receptors specially $\in \mathrm{n}$ dowed with the property. of being affected by hydrogen-ion concentration and able to convert this form of energy into nerve impulses. We have no histological evidence that any such receptors exist, but they cannot be excluded.
Unconditioned and Conditioned Reflexes.

There is good reason for denying the automaticity of nerve cells in that part of the central nervous system which Hughlings Jackson termed the "lower or first level." Hughlings Jackson defined this level as consisting of spinal cord, medulla, pons, and of the grey matter in the floor of the aqueduct of Sylvius. We know from the researches of Sherrington that this level, if cut off from the levels above, is capable of reflex action only. The reflexes it exhibits are in the decerebrate animal "unconditioned"; that is to say, they take place in a definite or predictable form which is dependent only upon the nature and site of the stimulus applied at the periphery.

The conditions which exist when the higher levels are not cut off are infinitely more complex. Then we have to deal not only with the reflex but with the various forms of sensation and with voluntary action. Many of the reflexes in the normal animal are transferred from the lower to the higher levels and become correspondingly more complicated. It is true not only of the reflexes of voluntary muscles but even of some of the visceral reflexes. The flow of saliva, when excited by the presence of suitable food in the mouth, is an "unconditioned" reflex. But Pavlov has shown that the reflex secretion of saliva may take place in response to many different forms of stimulus, even of a painful character, if these have been regularly associated with the presentation of food. Thus the ringing of a bell, or the electrical stimulation of a certain area of the skin, when regularly associated with the giving of food, become effective in themselves in provoking the reflex. This reflex Pavlov described as a "conclitioned" one, and the afferent paths of the nerve impulses which produce it must lead through the higher centres of the cerebral cortex, where it is liable to various forms of inhibition and is therefore no longer " unconditioned." The complexity of the reactions which take place in the cerebral cortex is partly due to the enormous number of pathways existing there, so that it is impossible to predict the reactions which any afferent impulse may cause unless such afferent path has been " conditioned" and all other afferent impulses are for the time being rigidly excluded. But the complexity of the cerebral reactions is also due to the exercise of the property of inhibition, which is a striking characteristic of the cerebrum, and appears to take place at the synapses. The cerebral cortex can direct the afferent impulses into any cf the efferent paths, but the choice of many of the latter must largely depend upon the connexions made by the pathways of the incoming nerve impulses.

\section{Sensation.}

Under normal conditions the nerve impulses which give rise to sensations have their origin outside the central nervous system, and are excited by the response of the sensory nerve endings or receptors to certain adequate forms of stimulation. The nerve impulses thus initiated are carried by afferent neurones to the grey matter of certain parts of the cerebral cortex or basal ganglia. Their further translation into consciousness is effected by some process the nature of which is unknown. The afferent impulses in their passage upwards may excite reflexes at various levels, and may or may not give rise to sensations. The production of sensation depends partly upon the anatomical pathways the nerve impulses take, but also upon the receptivity of that part of the brain which is concerned in translating them into terms of consciousness. The afferent paths utilized in the production of sensation are, in part at least, the same as those which are used in the afferent side of the conditioned reflex, and a sensation or a reflex, or both sensation and reflex, may result from the same afferent impulse. In states of excitement or mental stress a stimulus, which under ordinary conditions would givo rise to sensation or even pain, may set into activity the reflex mechanism but may fail to excite sensation, probably because the higher pathways of the cortex are fully occupied and by a process of inhibition the afferent impulses are prevented from exciting consciousness.

The Phystological Unit.

It is not customary to regard the sensation as a reflex, but the pathways of the nerve impulses which give rise to 
sensation are identical with those which are concerned on the afferent side with the production of Pavlov's " conditioned" reflex. The difference between the sensation and the reflex really lies in the nature of the process which produces the end-results. A sensation is a physiological or functional unit of the nerrous system just as a reflex is, and if the part of the brain which is responsible for the processes underlying consciousness be regarded as an effector the sensation becomes a reflex. From the clinical standpoint the sensation is as important as the reflex, and the causes which prodace disturbance in the one may produce disturbance in the other, and the manner of production of the disturbance is in large part the same in both. The term " reflex," though a very elastic one, has a definite meaning, and there are difficulties in classifying sensation under it. A new term for the physiological or functional unit of the nervous system which would embrace sensation, reflex, and voluntary movement might be better. It is this physiological or functional unit of the nervous sýsten which is affected and gives rise to the early manifestations of ill health.

Voluntary Movement.

The cerebral cortex gives origin to the impulses which bring about the contraction of voluntary muscle. The dendrites and bodies of the neurones concerned, the cells of Betz, which constitute the first neurones of the efferent chain, lie in the precentral convolution of the frontal lobe. There is no reason to suppose that the cells of Betz are automatic; they must be set into activity by nerve impulses reaching them from some other neurone. In the production of voluntary movement the nerve impulses pass from the cortex by the first efferent neurone into the lower level of the central nervous system, where they then make use of the efferent pathways of the lower or spinal reflex mechanism. The cells of Betz may be looked upon as part of a long reflex aro the efferent part of which begins in the cerebral cortex and extends to the lower level of the central nervous system. The cells of Betz may be set into activity by nerve impulses which reach them through the long afferent paths involved in the production of sensations. This form of activity may become a reflex taking place through the cerebral cortex, and is comparable with Pavlov's "conditioned" reflex, though its end-result is seen in voluntary muscle. Many of the complex movements make use of these long pathways. Speech, reading aloud, writing to dictation, the playing of musical instruments, and skilled movements generally, afford examples of voluntary action which have many of the characteristics of reflexes. Initiated voluntarily by some unknown process involving cerebral activity they may lose their voluntary character, and when the pathways for the nerve impulses have been cultivated by use the process becomes a reflex one. Some of the responses may be continued by a shortcircuiting as it were from the afferent to the efferent side at intermediate or lower levels. But in man the provision for even the simpler reflexes tends to be promoted to the higher levels. In injuries to the human spinal cord involving complete severance from the higher levels the part of the cord below the lesion fails at first to act as a reflex centre and only recovers as such when the direct pathways from the afferent to the efferent side in it have been reeducated.

Recipracal Innervation.

The contraction of one muscle or of one group of muscles cannot take place without affecting the state of other muscles. When the muscles which produce flexion at a joint are contracted the muscles which oppose the movement, the extensors, are stretched. In a reflex or voluntary contraction of muscles, exciting a definite act sueh as flexion, provision is made in the central nervous system for a twofold action. The stimulus which excites the discharge of nerve impulses by the efferent neurones supplying the flexor muscles also inhibits the activity of the efferent neurones supplying the opposing extensor muscles. There is indeed an actual elongation of the opposing muscles due to their sudden loss of tone. A reflex or voluntary contraction of one group of muscles is not a complete manifestation of the end-results of the nervous process concerned, for the nervous system is so arranged that muscle has a reeiprocal innervation. The phenomena of reciprocal innervation, the contraction of one group $c f$ muscles and the relaxation of another group, may be occasioned by nerve impulses coming from the higher levels as in voluntary movement, or by impulses entering the lower level of the central nervous system by afferent neurones from the periphery as in reflex movement.

Changes in the tension of each muscle excite receptors - the muscle spindles-in the muscle, and afferent nerve impulses are carried to the central nervous system, where they are co-ordinated and distributed to efferent neurones, stimulating some and inhibiting others, and thus leading to alterations in the tone of the muscles supplied.

The contraction of muscle alters the relative position of the paits of the body to one another. A constant stream of nerve impulses is carried into the central nervous system by afferent neurones from the proprioceptors, the receptors which are acted on by gravity and movement-for example, the utricle, saccule, and semicircular canals-and the receptors acted upon by tension-for example, the muscle spindles, and nerve endings in joints, ligaments, and other structures. Many of these impulses appear to be directed into the cerebellum, which Sherrington terms the head gangtion of the proprioceptive system. The impulses are co-ordinated in the cerebellum and distributed from it to efferent neurones in the lower level of the nervous system, and lead to alterations in the postural tone of the skeletal muscle.

The contraction of muscle, whether voluntary or reflex, is not an isolated act, but is accompanied by numerous complicated events in the central nervous system. The changes in muscle tone brought about by reflex action are of far-reaching importance, but are as yet imperfectly understood. Evidence is accumulating that the nervous processes concerned in the production of tone are in some respects different from those that have to do with ordinary contraction. The metabolism appears to be of a different character in the two events, and it is possible that the autonomic system plays a part in the maintenance of tone and of continued or tonic contraction of voluntary muscle, and that the actual contraction in the muscle is of a different nature.

Reciprocal innervation is not confined to voluntary muscle, but occurs in the innervation of all forms of muscle, and is shown by the heart and by the blood vessels. There is a close correlation of these organs by the nervous system. The contraction of voluntary muscle involves changes in the rate of the heart, the distribution of blood in the blood vessels, and alterations in metabolism. The liver liberates more glueose from its glycogen, and a number of changes take place in the chemistry of the body. The body is a unit and all parts work together. No change can occar in any of its parts without the production of some effect upon the others and upon the unit as a whole. If one examines the ascending columns of white matter in the lower level of the central nervous system one cannot but remark upon the great preponderance of neurones which are not associated with sensation, and which must be concerned in the production of reflexes. The central nervous system must be in constant receipt of afferent nerve impulses from all parts of the body, many of which have no direct representation in consciousness. Alterations in these impulses bring about changes in the body of a reflex nature. The altered reflex may in turn have a seeondary effeet npon consciousness. Reflexes involving alterations in metabolism, in the rate of the heart, in the distribution of the blood, and in respiration, to mention only the more obvious reflexes, may excite changes in conseiousness which give knowledge to the individual that all is not well, though the natrre of the sensation is vague and its actual cause is not recognized.

\section{Health and Ill Health.}

In the St. Andrews Institute we assume that health consists in the harmonious working together of all the organs and tissues of the body. The process by which this is effected is a nervous one of great complexity. A state of health implies that all parts of the body are furnishing from the various receptors their normal quota of afferent 
nerve impulses to the central nervous system. These in turn give rise to normal responses which are sensations and reflexes. The basis of the process is the physiological or functional unit of the nervous system.

Ill health, on the other hand, reveals itself by some disturbance of this process, and we are seeking to recognize the significance of these disturbances. Ill health is not necessarily associated with any visible pathological change in the organs of the body. When the nervous processes are disturbed the fact is usually recognized by the patient before any change in the organs can be demonstrated by the pathologist. Alterations in sensation occur which the patient recognizes as abnormal, and such alterations may be the first indication of the departure from normal health. Concurrently there may be reflex phenomena which show themselves as definite symptoms though they may be diffcult to recognize. Any part of the reflex arc, or of the anatomical basis of the physiological unit, may be the site of the disturbance, though some parts of it are more susceptible than others.

Our contention is that we must look for the first signs of ill health in the nervous regulating processes of the body. They are the basis of the vital activities and maintain the life of the organism as a unit. Disturbance of the vital processes is the first sign of ill health, and their failure is the ultimate cause of death.

\section{SOME CAUSES OF INTESTINAL OBSTRUCTION.*} BY

\author{
J. W. THOMSON, M.A., M.B., C.M., \\ SURGEON, CLAYTON HOSPITAL, WAKEFIELD.
}

\section{I.-Volvulus of the Small Intestine.}

Volvulus of the small intestine is a very rare condition, and the whole of the jejunum and ileum may be involved, or only one loop may be affected. Tully Vaughan, as quoted in Rowlands and Turner's Operations of Surgery, has collected 21 cases. In several of these the difficulties were so great, and the appearances so puzzling, that the operators did not recognize the condition during operation. The following is a case of volvulus of the small intestine :

A man, aged 23, was admitted to a nursing home in November, 1920, about midnight, after a journey of about forty miles by road in an open touring car. Forty-eight hours previously he had been seized with violent pain in the abdomen, round the umbilicus, accompanied by vomiting. The pain never entirely subsided, but hs had violent attacks at intervals. He had vomited everything, and had passed no flatus. On admission he was in a very weak condition-temperature $97^{\circ}$, pulse 120 . During the examination of the abdomen peristalsis was excited, and a coil of bowel could be felt below and to the right of the umbilicus

The abdomen was opened through the right rectus, and there was a considerable escape of sanguineous fluid. Bands and adhesions were present in puzzling perplexity. Coils of and intestine were adherent to the lateral and anterior abdominal walls and to the caecum, obscuring this strueture, and to each other. During the separation of these bands and adhesions, what was momentarily thought to be another stout and strong band was revealed, which, from its size (rather larger than a lead pencil) and appearance, and pathological associations, almost invited resection. On tracing this towards the caecum it terminated abruptly one inch from the ileo-caecal junction, at the point where a string band had bound the intestine to the abdominal wall. It passed downwards and inwards for four or five inches, terminating in slightly distended intestine, and it was now obviously a volvulus of the ileum. This was easily unfolded, and there were about three turns. The intestine rapidly regained its colour and peristaltic movement. The raw surfaces were sutured, or covered with omental grafts. The patient made were uninterrupted recovery.

The patient was a strong, athletic man, who had had an attack of appendicitis seven years previously, when he was ill for six or sutured or covered with omental grafts. The patient made an quite well in the interval.

The case was of interest because of its rarity and its primary cause, and also of the long interval between the cause and effect. It is condemnatory of the expectant treatment of appendicitis, and it reminds one that after such an attack there may lurk in the abdomen unsuspected

\footnotetext{
*A paper read before the Yorkshire Branch of the British Medica!
}

forces that, given favourable conditions, even after the lapse of many years, are potential of swift calamity. A perhaps simple appendectomy is replaced by a difficult and dangerous operation.

\section{II.-Dudedenal Hernia.}

There are two varieties of duodenal hernia-right and left-the left being the more common. The left hernia originates in the paraduodenal fossa of Landzert. Trietz states that three conditions are necessary for the formation of a left duodenal hernia:

\section{The existence of a fossa and its boundary folds.}

2. The presence of the inferior mesenteric vein in the fold.

3. Sufficient mobility of the small intestine to permit its entry into the sac. The mouth of the sac may be two or three inches wide. The size of the hernia varies from that of a walnut to the size of a man's head, and may contain all the small intestines. The length of intestine may be as small as two inches.

Left duodenal hernia occupies at first the left and upper portion of the abdomen, but eventually may occupy nearly the entire cavity. In 1899 Moynihan collected 57 cases of left duodenal hernia. Diagnosis is rarely made, and the condition is discovered at operation for acute intestinal obstruction or at autopsy.

The following is a case of left duodenal hernia in a woman :

History.-Thepatient was healthy as a child. Her first illness was acute rheumatism at the age of 21 . She was ill for three months, but eventually made a good recovery. Since that attack, until June, 1920 , she enjoyed very good health, but was inclined to be constipated at times. She was not in the habit of taking aperients. In June, 1920, she had a slight accident, falling backwards over the foot of a bed, and she had had soreness and stiffness in her back since. Immediately after each meal, and without fail, she pubic region. The pain lasted about fifteen minutes, disappearing entirely. Coincident with the pain was distension of the abdomen, the latter disappearing at the same time as the former. Since the accident she had been more constipated, and had been compelled accident she had been more constipated, and had been compelled to take aperients on alternate days, but she never vomited.
This condition persisted until November 8th, 1920, when she This condition persisted until November 8th, 1920, when she dinner, passing away in the usual time. About three-quarters of an hour afterwards she was seized with violent pain in the epigastrium, causing her to sink to the floor and roll about in agony. She had no vomiting on that occasion. The pain persisted for about two and a half hours, gradually disappearing. She slept well the following night. On November 9th, 10th, and 11 th, though she had slight continuous nausea, but no vomiting. Her diet was restricted to milk, and she noticed slight abdominal distension. The only actions of the bowels during this period were several watery motions on November 9th, after taking castor oil. On the 12 th another acute attack set in about noon. This time
the pain persisted, abdominal distension became very marked, she the pain persisted, abdominal distension became very
vomited very persistently, and no flatus was passed.

Condition on Admission. - She was admitted to hospital the same Condition on Admission.- She was admitted to hospital the same
evening about 10.30. Abdominal distension was marked. The abdomen was tender, and the pain very severe. The pulse was imperceptible at the wrist, and collapse was intense. Vomiting had ceased. Immediate operation was decided upon, and under the anaesthetic palpation revealed 2 large abdominal tumour, resembling an ovarian cyst, though obviously not of pelyic origin. It was slightly movable, but not on respiration, and resonant on spinous line. My house-surgeon remarked that the outline of the

abdomen was that of an advanced pregnancy.
Operation.- The abdomen was opened in the middle line, and Operation.- The abdomen was opened in the middle line, and
there was revealed a large, smooth, glistening tumour. No intesthere was revealed a large, smooth, glistening tumour. No intes-
tine presented itself, nor did any sanguineous fluid escape at this tine presented itself, nor did any sanguineous fluid escape at this
stage. The hand was passed round the tumour, beginning on the stage. The hand was passed round the tumour, beginning on the
right side. On reaching the lower border, and on a level with the third lumbar vertebra, an orifice was discovered admitting easily a thumb and two fingers. Between the orifice and the caecum were a few inches of collapsed ileum, with haemorrhages in its wall and mesentery. There was no entering loop of intestine, only the retiring segment. With the gentlest traction the intestine was drawn from the sac. About mid-jejunum there was a well marked constriction ring, and for many inches on either side of this the intestine was black but viable. Blood-stained fluid escaped from the sac along with the intestine. No attempt patient. Though desperately ill for a day or two the patient made a rapid recovery.

Operation was undertaken with the hope that some simple manipulation, rapidly executed, might still avert death that was imminent, and in this respect it was fortunate that the intestine was withdrawn without the 